\title{
A Comprehensive Characterization of Particulate Matter, Trace Elements and Gaseous Emissions of Piston-Engine Aircraft
}

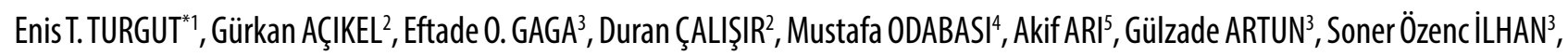
Umut SAVACl ${ }^{6}$, Emre CAN ${ }^{3}$, Servet TURAN ${ }^{6}$

' Eskisehir Technical University, Faculty of Aeronautics and Astronautics, Aircraft Airframe and Powerplant Department, Eskisehir, TURKEY

${ }^{2}$ Eskisehir Technical University, Aircraft Maintenance Centre, Eskisehir, TURKEY

${ }^{3}$ Eskisehir Technical University, Faculty of Engineering, Department of Environmental Engineering, Eskisehir, TURKEY

${ }^{4}$ Dokuz Eylul University, Faculty of Engineering, Department of Environmental Engineering, Izmir, TURKEY

${ }^{5}$ Bolu Abant Izzet Baysal University, Faculty of Engineering, Gölköy Campus, Bolu, TURKEY

${ }^{6}$ Eskisehir Technical University, Faculty of Engineering, Department of Materials Science and Engineering, Eskisehir, TURKEY

\section{SUPPORTING INFORMATION}

\section{ADDITIONAL EXPLANATION}

High power engine run S1

Sampling volumetric flow ...S1

RGB channels

\section{FIGURES}

Figure S1. Filters and $\mathrm{ACl}$ values of all tests...

Figure S1. Continued.

Figure S2. Variation of ACl with main engine parameters. ...S4

Figure S3. Variation of PM mass concentration with engine speed under different fuel mixture ratios (a-c) ........................................S5

Figure S4. Variation of PM mass concentratiton with main engine parameters.

Figure S5. STEM-EDX spectra (a-k) obtained from the PMs. Labels used in this figure correspond to same labels given in Figure 10. In the $(i, k)$, spectra are zoomed and given as an inset to obtain better visibility of $F e$ and $\mathrm{Cr}$ elements. ... 57

Figure S6. EDX spectra taken from particles that contain Al element in its chemical composition. The spectras (a), (b) and (c) were taken from the particles given in Figure $10(\mathrm{~g})$, $(\mathrm{h})$ and $(\mathrm{i})$, respectively.

\section{TABLES}

Table S1. Description of FLO analyses .59

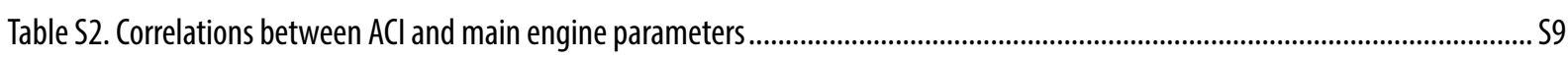

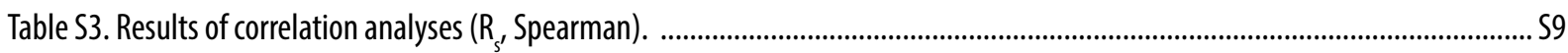

Table S4. FLO analyses results ranked in order of the highest-to-lowest values at fuel samples. ......................................................... S10 


\section{High power engine run}

It can be seen from Figure 2 that the horizontal axes involve two additional speed points, 2250 and $2500 \mathrm{rpm}$, to show observations under the BP mixture. During ground operations, we carefully experimented with $2250 \mathrm{rpm}$ for threeminutes and $2500 \mathrm{rpm}$ for one-minute under the BP mixture, where we were only able to measure the gaseous emissions. This was not included in the test matrix, since the engine manufacturer does not recommend high power runs on the ground due to an overheating risk. After this experiment, we decided not to run the engine above 2000 rpm because of the elevated temperature of lubrication oil and engine case. Since there was only one measurement at both speed points, we were not able to obtain repeatable results and, therefore, we did not present any emission results at these speed points. On the other hand, we kept the engine data related to these speed points, in Figure 2, since they were directly obtained from the aircraft indication system.

\section{Sampling volumetric flow}

While the sampling flow rate of the unit was set to a constant value of $20 \mathrm{~L} \mathrm{~min}^{-1}$ and a duration of 5 minutes, there may be small variations in the sampled exhaust gas volume $\left(\mathrm{V}_{\mathrm{d}}\right)$ under real world conditions. Therefore, although the expected $\mathrm{V}_{\mathrm{d}}$ is $100 \mathrm{~L}$, descriptive statistical analysis results indicate an average of $98.1 \pm 1.7 \mathrm{~L}$ (ranging between 90 and $102 \mathrm{~L}$ ) at all of the tests. While all of the mass based PM concentrations can be adjusted with respect to the actual $V_{d}$, these small variations might be considered to be a source of error for the Accumulation Color Index (ACI), a parameter introduced in the following paragraphs, since lower $\mathrm{V}_{\mathrm{d}}$ tends to a lower accumulation. However, there was no correlation found between the actual $\mathrm{V}_{\mathrm{d}}$ and ACI parameters. One explanation for this could be due to the fact that considering the 5 minutes sampling time-frame, variations at this level in $\mathrm{V}_{\mathrm{d}}$ do not cause any considerable change in accumulation which create a distinguishable tone color change.

\section{RGB channels}

The RGB color mode involves three channels (primary colors), namely, red, green and blue. ${ }^{1}$ Having 8 bits per channel, there are total of 24 bits of color information for each pixel of a color image. The color intensity of each primary color can be stored in a range between 0 (black) and 255 (white). This means that an image can be displayed using more than 16.5 million $(255 \times 255 \times 255)$ different color tones. ${ }^{1}$ In this context, a red color channel provides the intensity of red color in a range between 0 and 255 as a gray scale of this single color. Being closer to 255 indicates a higher intensity of red color while 0 means there is no red color. Finally, color channels could be good indicator to estimate, for instance, leaf flushing (green) and senescence (red) ${ }^{2}$ or trace element concentration. ${ }^{3}$ However, this is beyond the scope of this study and the ACI values are obtained directly from the descriptive statistical results of the software as a combination of three channels.

\section{References:}

(1) Adobe, https://helpx.adobe.com/photoshop/using/color-modes.html (Access: 09 April 2020).

(2) Alberton, B.; Torres, R. S.; Cancian, L. F.; Borges, B. D.; Almeida, J.; Mariano, G. C.; Santos, J.; Morellato, L. P. C. Introducing Digital Cameras to Monitor Plant Phenology in the Tropics: Applications for Conservation. Perspectives in Ecology and Conservation. 2017, 15 (2), 82-90. https://doi.org/10.1016/j.pecon.2017.06.004.

(3) Hatiboruah, D.; Das, T.; Chamuah, N.; Rabha, D.; Talukdar, B.; Bora, U.; Ahamad, K. U.; Nath, P. Estimation of Trace-Mercury Concentration in Water Using a Smartphone. Measurement. 2020, 154, 107507. https://doi. org/10.1016/j.measurement.2020.107507. 


\section{Engine speed (RPM)}

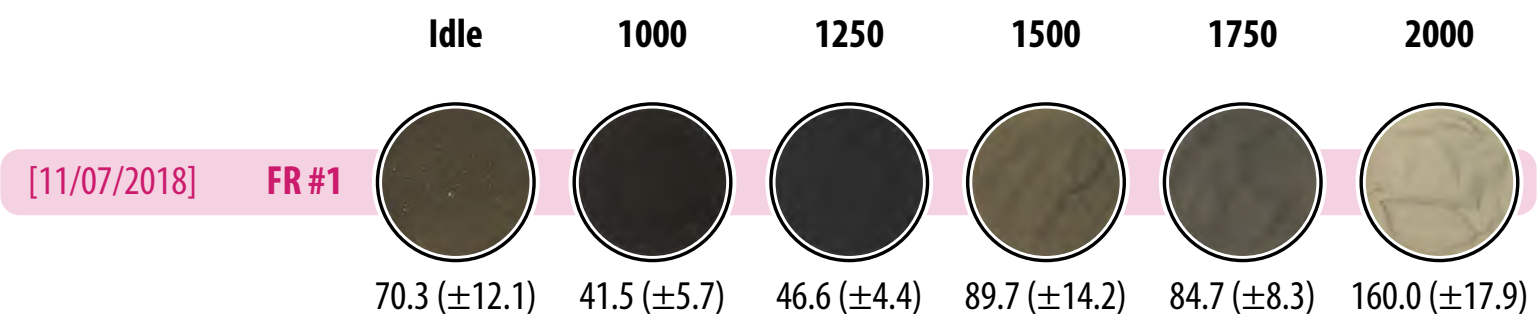

\section{[24/07/2018] FR \#2}
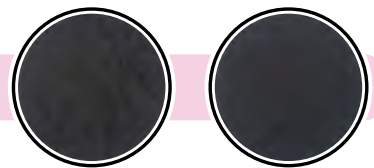

$\otimes$

$\otimes$

$\otimes$

$\otimes$

$55.7( \pm 7.4) \quad 56.1( \pm 6.5)$

\section{$[24 / 07 / 2018]$ \\ FR \#2}
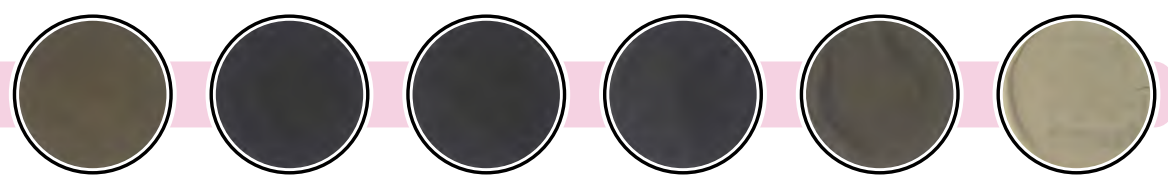

$86.4( \pm 10.3)$

$60.0( \pm 5.3)$

$60.5( \pm 5.4)$

$65.9( \pm 6.2)$

$84.0( \pm 8.7)$

$150.8( \pm 16.4)$

\section{[24/07/2018] BP\#2}
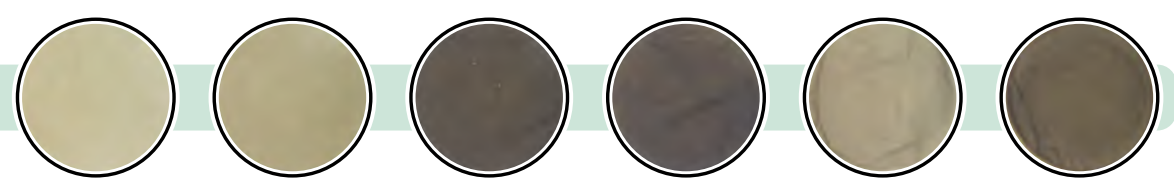

$182.9( \pm 18.0) \quad 159.0( \pm 19.7)$

$88.9( \pm 8.1)$

$91.8( \pm 8.6)$

$152.1( \pm 17.3) \quad 98.2( \pm 17.3)$
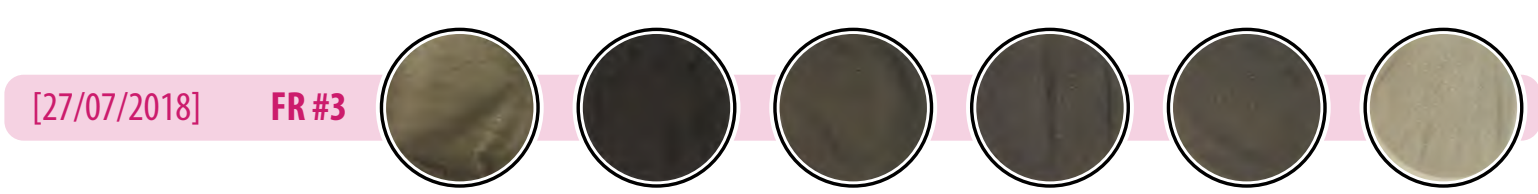

$92.1( \pm 22.4)$

$46.1( \pm 5.3)$

$70.3( \pm 8.9)$

$74.6( \pm 8.5)$

$78.8( \pm 9.6)$

$157.1( \pm 18.9)$
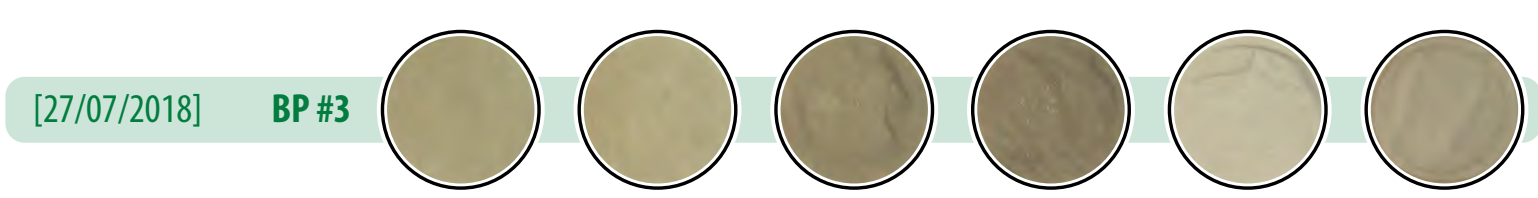

$149.3( \pm 20.1) 158.0( \pm 20.9) \quad 129.7( \pm 19.0) 121.0( \pm 18.3) \quad 179.9( \pm 15.9) \quad 147.8( \pm 18.6)$

Fig. S1 Filters and ACI values of all tests 


\section{Engine speed (RPM)}

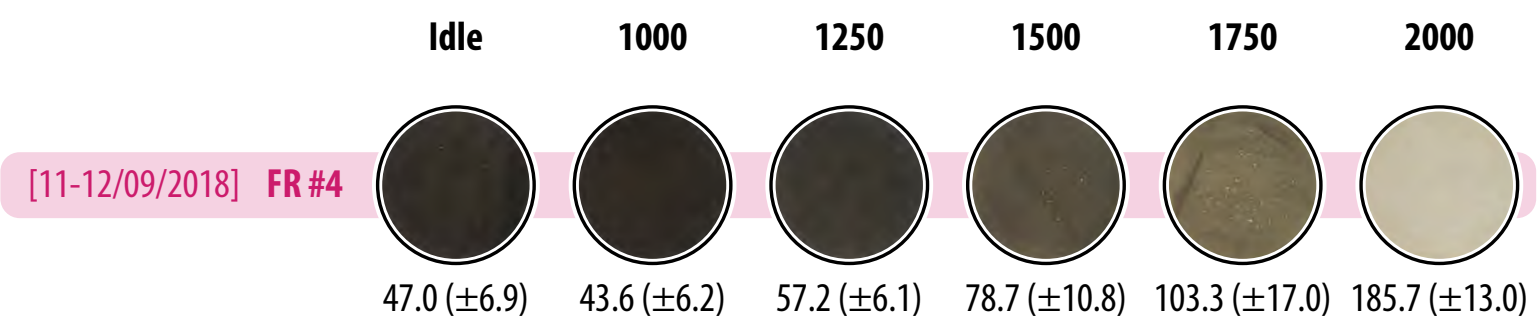

[11-12/09/2018] BP\#4
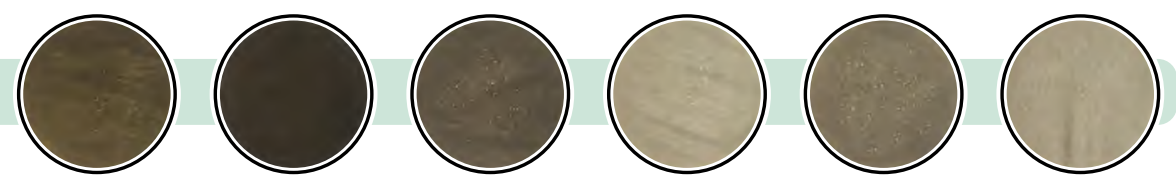

$75.4( \pm 17.4)$

$51.1( \pm 8.2)$

$84.7( \pm 12.7)$

$156.9( \pm 17.1) 113.5( \pm 15.7) 153.2( \pm 16.1)$

[11-12/09/2018] BE\#4
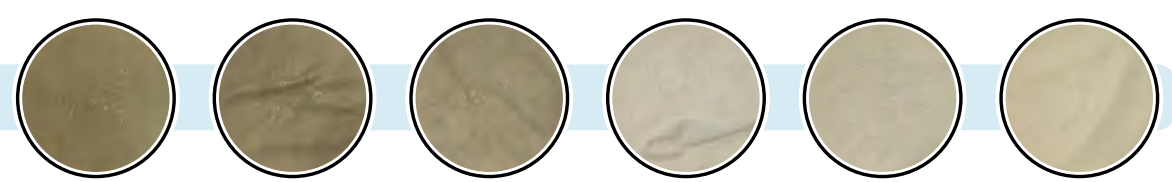

$115.5( \pm 21.0) 118.1( \pm 19.7) 135.9( \pm 18.1) \quad 183.5( \pm 12.4) 176.5( \pm 13.7) 181.0( \pm 16.8)$

\section{[3-4/01/2019] FR\#5}
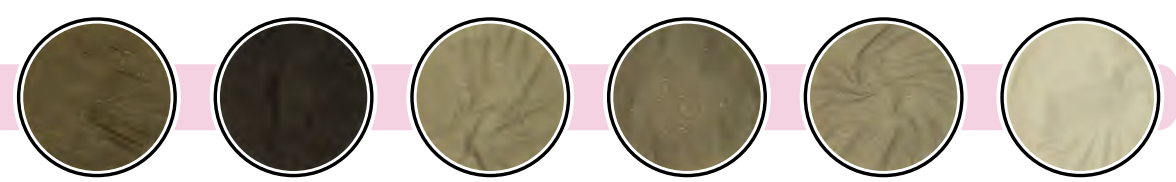

$73.8( \pm 19.1)$

$38.6( \pm 8.3)$

$124.9( \pm 21.0) 101.9( \pm 18.2)$

$127.9( \pm 19.6) 186.5( \pm 16.9)$

\section{[3-4/01/2019] BP\#5}
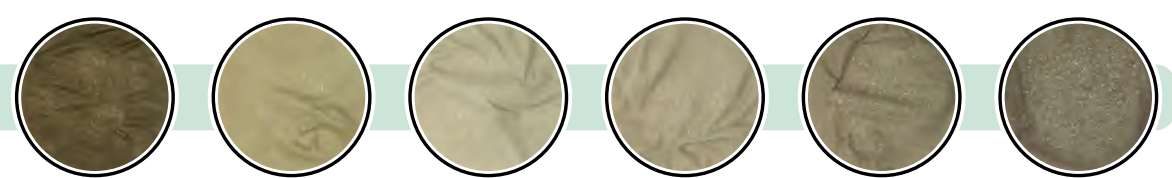

$77.9( \pm 20.6) \quad 148.8( \pm 23.7) \quad 167.3( \pm 19.1) \quad 151.1( \pm 18.6) \quad 121.9( \pm 18.9) \quad 110.2( \pm 18.6)$

\section{[3-4/01/2019] BE\#5}
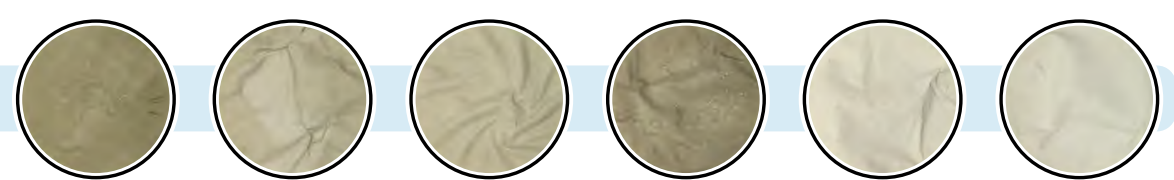

$130.6( \pm 20.9) \quad 167.7( \pm 19.6) \quad 170.8( \pm 17.8) \quad 131.4( \pm 20.0) 202.1( \pm 14.1) 200.5( \pm 11.1)$

Fig. S1 Continued 

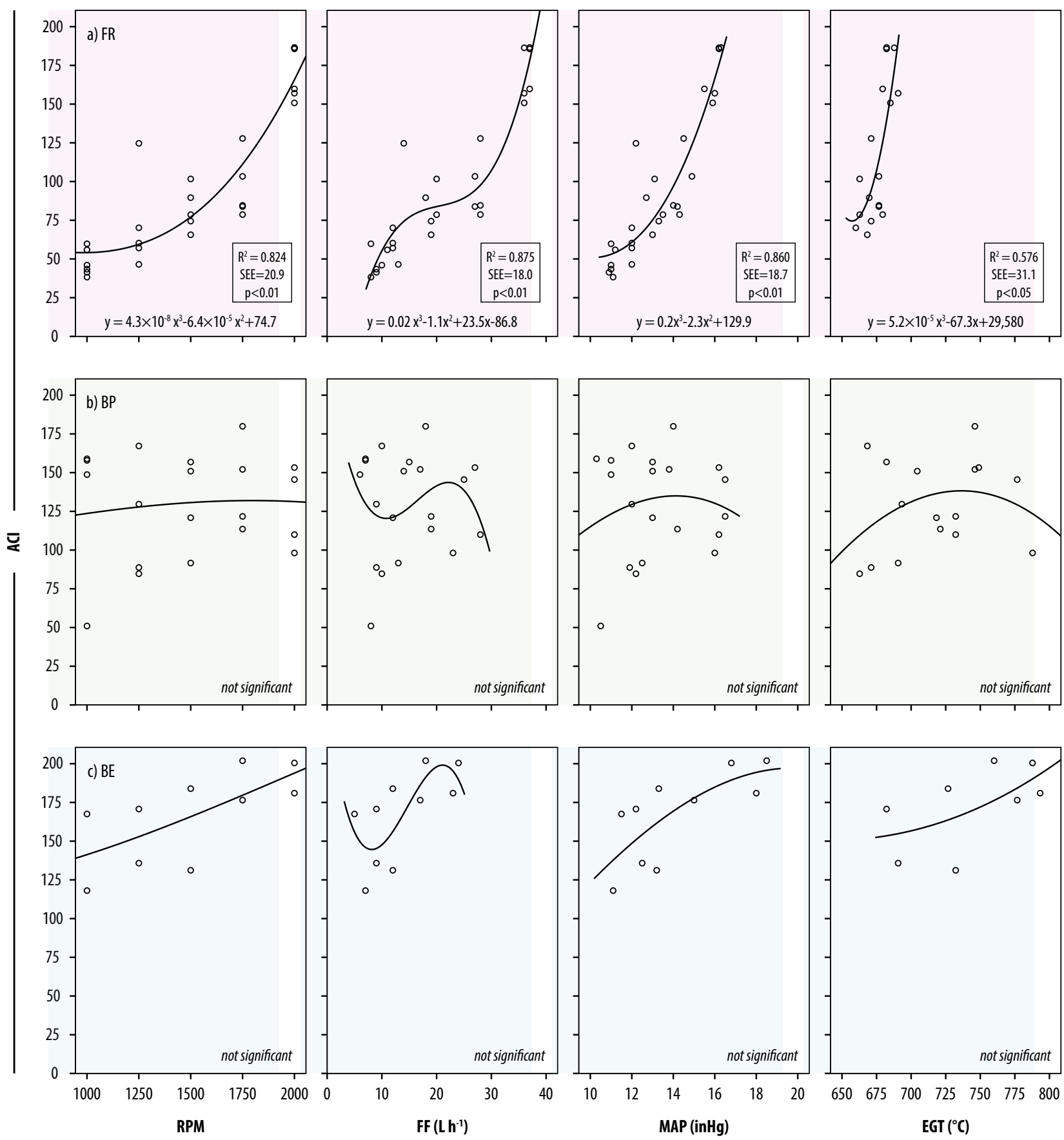

Fig. S2 Variation of ACI with main engine parameters. 

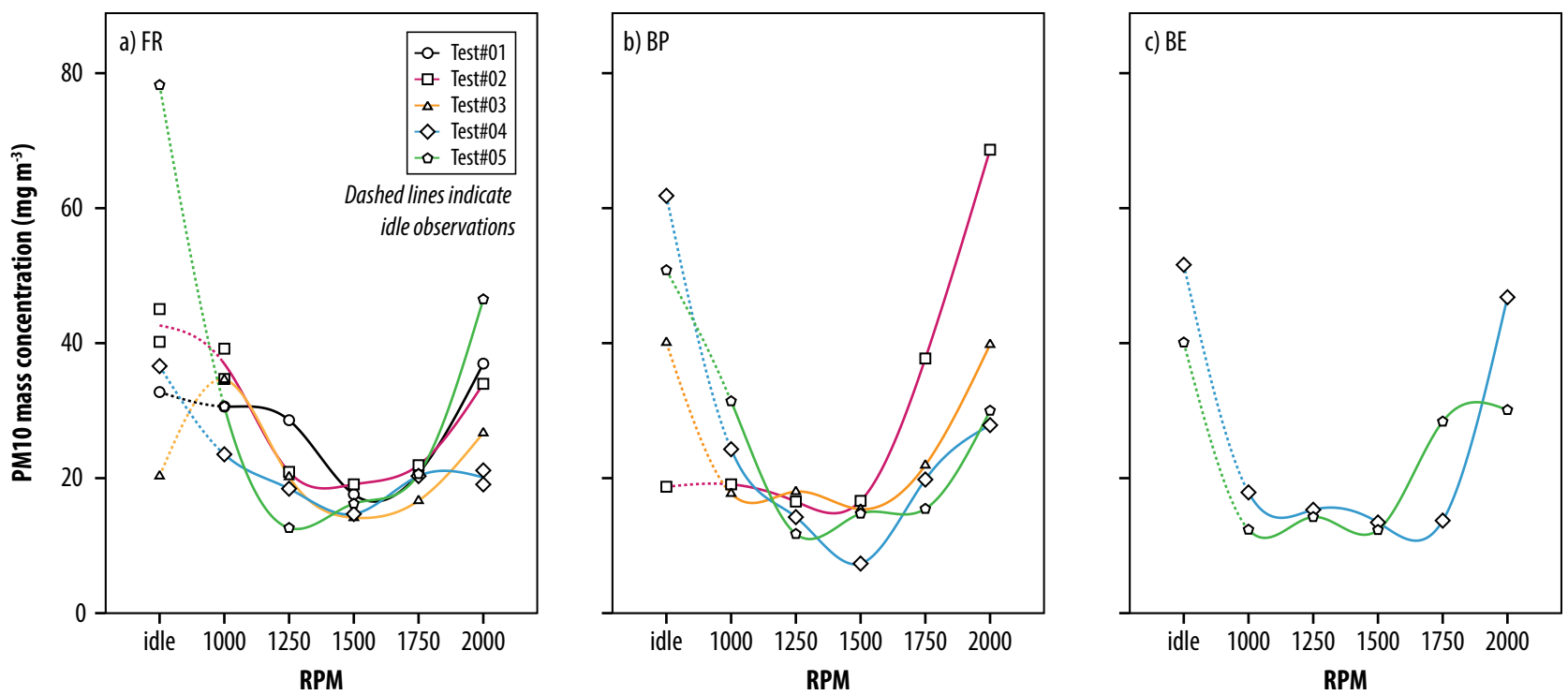

Fig. S3 Variation of PM mass concentration with engine speed under different fuel mixture ratios (a-c) 

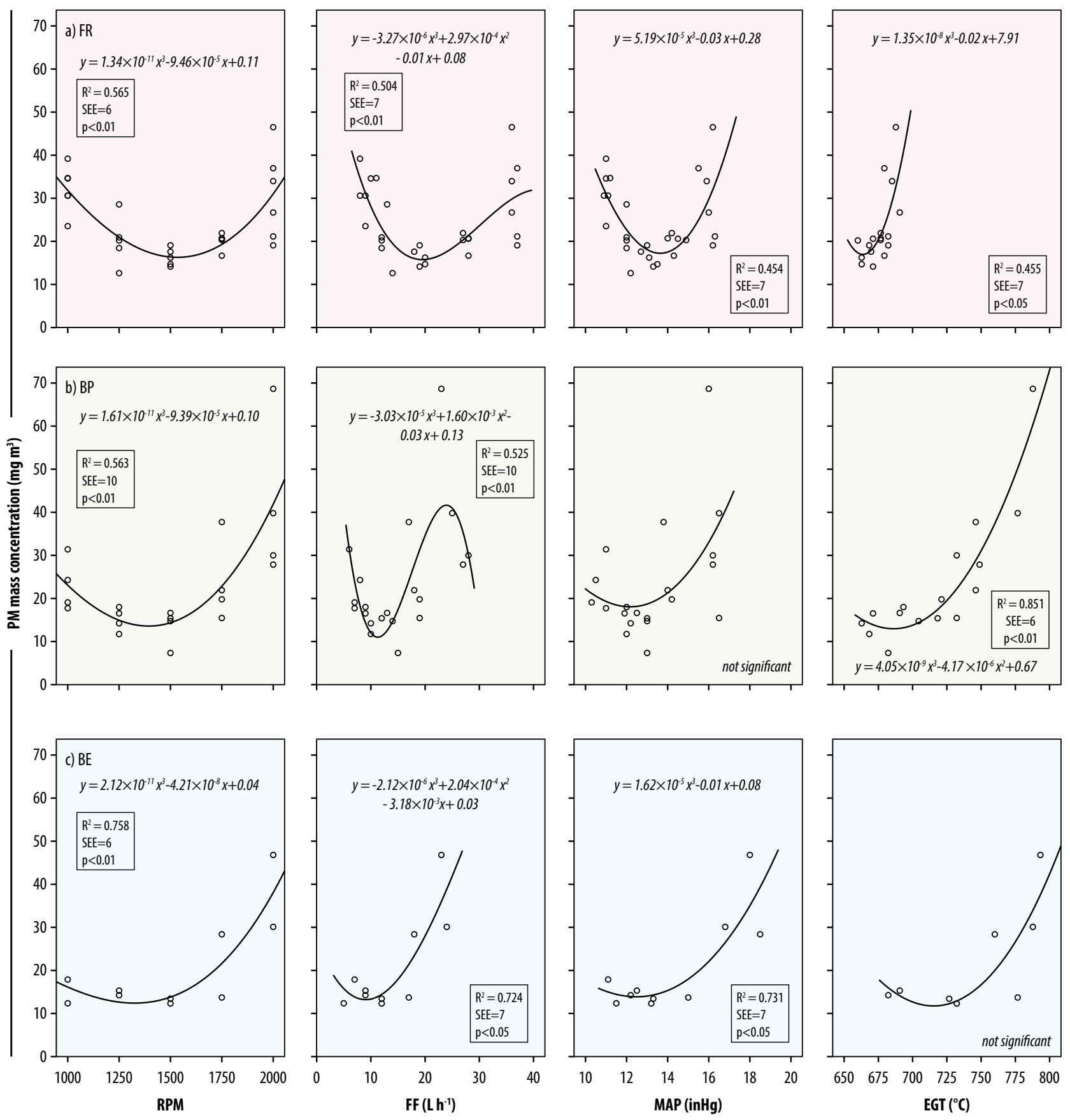

Fig. S4 Variation of PM mass concentratiton with main engine parameters. 


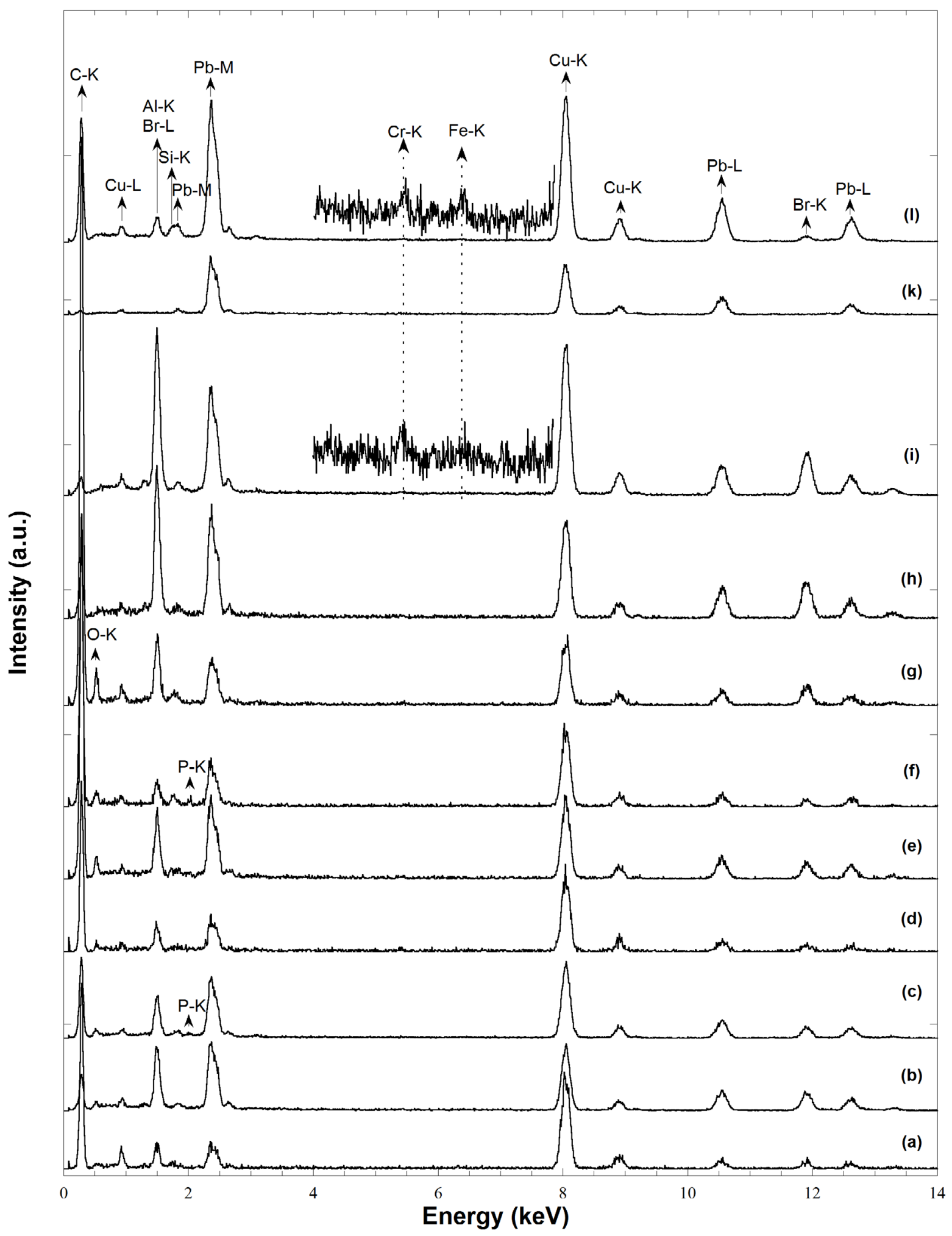

Fig. S5 STEM-EDX spectra (a-k) obtained from the PMs. Labels used in this figure correspond to same labels given in Fig. 10. In the (i, $k$ ), spectra are zoomed and given as an inset to obtain better visibility of Fe and Cr elements. 


\section{The method used for the Identification of Aluminum in EDX spectra:}

As it can be seen in the EDX spectra, Al-K (1.486 keV) and $\mathrm{Br}-\mathrm{L}(1.481 \mathrm{keV})$ peaks are overlapped and in order to identify whether $\mathrm{Al}-\mathrm{K}$ contributes to peak intensity or not at $1.48 \mathrm{keV}$, the intensity ratio between $\mathrm{Br}-\mathrm{K}$ and $\mathrm{Br}-\mathrm{L}$ peaks is used. To determine the value of (IBr-L/IBr-K), EDX spectrum of KBr sample was taken at the same TEM as well as same condition with analyses of PMs. According to the $\mathrm{KBr}$ spectrum the ratio between $\mathrm{K}$ and $\mathrm{L}$ peaks of $\mathrm{Br}$ is found as 3.5. According to this value presence of $\mathrm{Al}$ was determined as following procedure. Intensity of $\mathrm{Br}-\mathrm{K}$ peak at $11.924 \mathrm{keV}$ was measured and this value is multiplied with 3.5 to calculate Br-L peak intensity value. Then the peak intensity at $1.84 \mathrm{keV}$ is measured from the experimental EDX spectrum and it is compared with the calculated Br-L peak intensity value. If the measured value of $\mathrm{IBr}-\mathrm{L}$ is higher than the calculated value, it corresponds that $\mathrm{Al}-\mathrm{K}$ peak contributes to peak at $1.84 \mathrm{keV}$ besides $\mathrm{Br}-\mathrm{L}$ peak and the presence of Al element is confirmed. The figure given in Fig. S6 summarize the method describe given above. Red spectra is obtained from $\mathrm{KBr}$ sample and $\mathrm{Br}-\mathrm{K}$ peak is normalized to experimental $\mathrm{Br}-\mathrm{K}$ peak intensity.

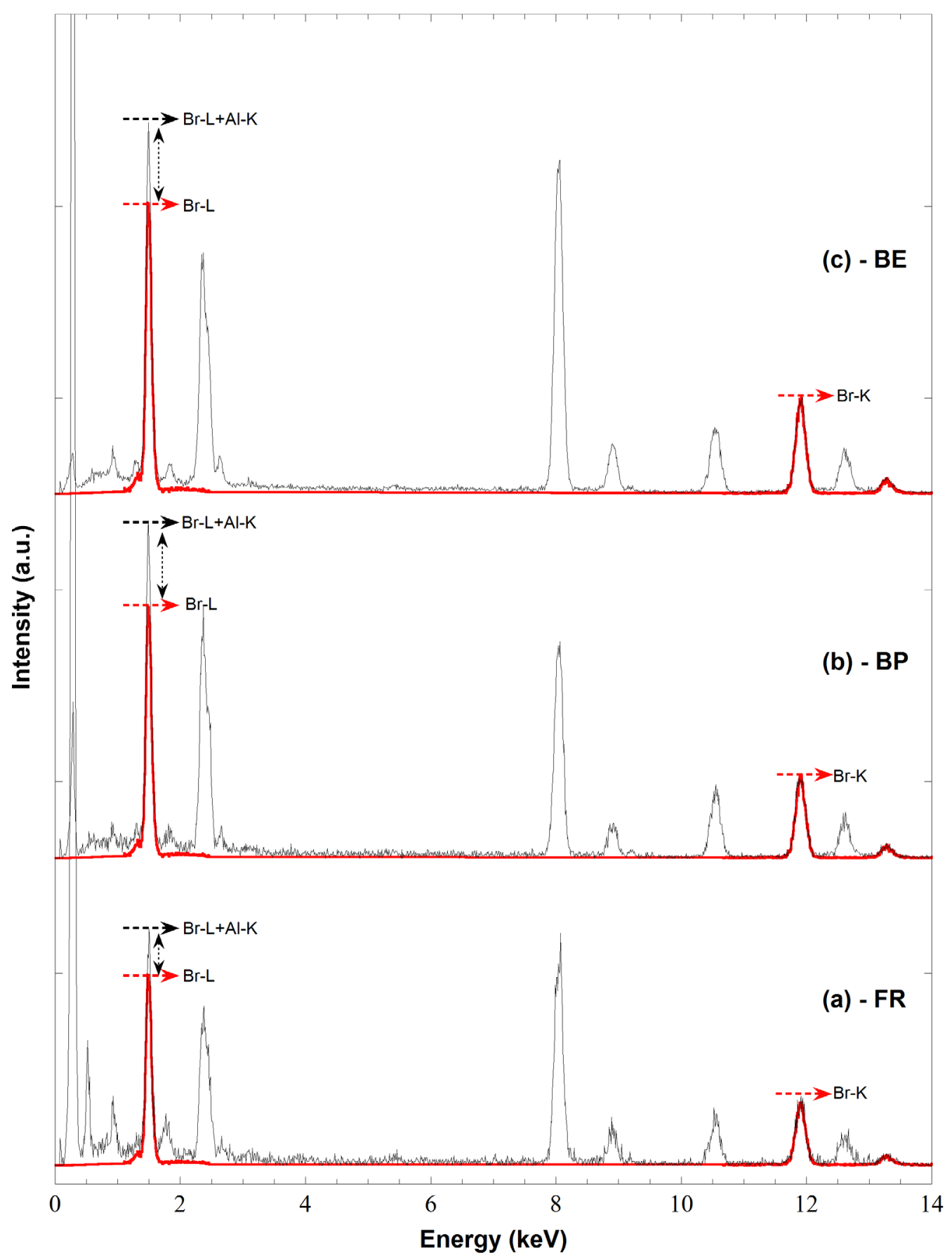

Fig. S6 EDX spectra taken from particles that contain Al element in its chemical composition. The spectras (a), (b) and (c) were taken from the particles given in Fig. 10 (g), (h) and (i), respectively. 


\begin{tabular}{llllc}
\hline No & Date & Aircraft & Engine & Brand name \\
\hline 01 & $08 / 03 / 2018$ & TB-20 & IO-540-C4D5D Exxon Elite \\
02 & $08 / 03 / 2018$ & AG-5B Tiger & O-360-A4K & Aeroshell 15W50 \\
03 & $08 / 03 / 2018$ & AG-5B Tiger & O-360-A4K & $"$ \\
04 & $08 / 03 / 2018$ & TB-20 & IO-540-C4D5D Exxon Elite \\
05 & $08 / 03 / 2018$ & Cessna 172S & IO-360-L2A & $"$ \\
06 & $08 / 03 / 2018$ & Cessna 172S & IO-360-L2A & $"$ \\
07 & $08 / 03 / 2018$ & Cessna 172S & IO-360-L2A & $"$ \\
08 & $08 / 03 / 2018$ & Cessna 172S & IO-360-L2A & $"$ \\
09 & $21 / 06 / 2018$ & TB-20 & IO-540-C4D5D & $"$ \\
10 & $30 / 07 / 2018$ & AG-5B Tiger & O-360-A4K & Aeroshell 15W50 \\
11 & $12 / 09 / 2018$ & TB-20 & IO-540-C4D5D Exxon Elite \\
12 & $08 / 03 / 2018$ & - & - & Exxon Elite- unused \\
\hline 01 & $08 / 03 / 2018$ & AG-5B Tiger & O-360-A4K & AVGAS LL100 \\
02 & $21 / 06 / 2018$ & TB-20 & IO-540-C4D5D & $"$ \\
03 & $30 / 07 / 2018$ & AG-5B Tiger & O-360-A4K & $"$ \\
04 & $12 / 09 / 2018$ & TB-20 & IO-540-C4D5D & $"$ \\
\hline
\end{tabular}

Table S2. Correlations between $\mathrm{ACl}$ and main engine parameters

\begin{tabular}{lcccccc}
\hline Engine & \multicolumn{3}{c}{ All engine speed } & \multicolumn{3}{c}{ Idle excluded } \\
\cline { 2 - 7 } parameter & FR (33) & BP (24) & BE (13) & FR (27) & BP (20) & BE (11) \\
RPM & $\mathbf{0 . 7 7 6 * *}^{* *}$ & - & $\mathbf{0 . 7 7 9}^{* *}$ & $\mathbf{0 . 8 6 0}^{* *}$ & - & $0.677^{*}$ \\
FF & $\mathbf{0 . 8 4 7 * *}_{\text {MAP }}^{* *}$ & - & $\mathbf{0 . 7 5 5}^{* *}$ & $\mathbf{0 . 8 9 0}^{* *}$ & - & $0.662^{*}$ \\
EGT & $\mathbf{0 . 8 3 7}^{* *}$ & - & $\mathbf{0 . 7 9 5}^{* *}$ & $\mathbf{0 . 8 9 9}^{* *}$ & - & $\mathbf{0 . 7 2 8}^{*}$ \\
\hline
\end{tabular}

Numbers inside parenthesis represent $\mathrm{N}$ under corresponding mixture ratio.

${ }^{*}: \mathrm{p}<0.05-{ }^{* *}: \mathrm{p}<0.01-$

Table S3. Results of correlation analyses ( $R_{s}$ Spearman).

\begin{tabular}{|c|c|c|c|c|c|c|c|c|c|}
\hline \multirow[t]{2}{*}{ Element } & \multicolumn{3}{|c|}{$\begin{array}{c}\text { Correlations with engine speed } \\
(\text { idle } \rightarrow 2000 \mathrm{rpm}) \\
\end{array}$} & \multicolumn{6}{|c|}{$\begin{array}{l}\text { Correlations with mixture ratio } \\
\qquad(\mathrm{FR} \rightarrow \mathrm{BP} \rightarrow \mathrm{BE})\end{array}$} \\
\hline & $\mathrm{BE}$ & BP & FR & idle & 1000 & 1250 & 1500 & 1750 & 2000 \\
\hline Al & - & - & - & - & - & - & - & - & - \\
\hline As & - & - & - & - & - & - & - & $-0.706^{\star}$ & - \\
\hline $\mathrm{Ca}$ & - & - & - & - & - & - & - & $-0.811^{\star \star}$ & - \\
\hline Cd & - & $0.465^{\star}$ & $0.522^{\star \star}$ & - & - & - & - & - & - \\
\hline $\mathrm{Cr}$ & - & $-0.546^{\star *}$ & - & - & - & - & - & - & - \\
\hline Ga & $0.847^{\star \star}$ & - & - & - & - & - & - & - & - \\
\hline Ge & - & - & $0.376^{\star}$ & - & - & - & - & - & - \\
\hline $\mathbf{L a}$ & - & - & - & - & $0.600^{*}$ & $-0.686^{\star}$ & - & - & - \\
\hline Mg & - & $-0.511^{\star}$ & $-0.449^{\star}$ & - & - & - & - & $-0.633^{\star}$ & - \\
\hline $\mathrm{Na}$ & - & - & - & - & - & - & - & - & $-0.580^{*}$ \\
\hline $\mathrm{Ni}$ & - & - & $-0.427^{\star}$ & - & - & - & - & - & - \\
\hline $\mathbf{P b}$ & $0.763^{\star *}$ & $0.599^{\star *}$ & $0.492^{\star \star}$ & - & $-0.672^{\star}$ & - & - & - & - \\
\hline $\mathbf{S}$ & - & - & $-0.514^{\star \star}$ & - & - & - & - & - & - \\
\hline Se & - & - & $0.376^{\star}$ & - & - & - & $-0.717^{\star}$ & - & - \\
\hline $\mathrm{Sr}$ & - & - & - & - & - & - & - & $-0.633^{\star}$ & - \\
\hline V & - & - & - & - & - & - & - & $-0.765^{\star}$ & - \\
\hline Zn & - & - & - & - & - & - & - & $-0.809^{* *}$ & - \\
\hline$\sum \mathrm{TEL}$ & $0.910^{\star \star}$ & $0.574^{\star \star}$ & $0.486^{\star *}$ & - & $-0.657^{\star}$ & - & - & - & - \\
\hline$\Sigma \mathbf{P M}$ & - & - & - & - & $-0.802^{\star *}$ & - & - & - & - \\
\hline$\sum \mathrm{TEL} / \Sigma \mathbf{P M}$ & - & $0.599^{\star \star}$ & $0.739^{\star *}$ & $0.588^{\star}$ & - & - & - & - & - \\
\hline
\end{tabular}

${ }^{*}:<0.05 ;{ }^{* *}:<0.01 ;-$ : not significant 
Table S4. FLO analyses results ranked in order of the highest-to-lowest values at fuel samples.

\begin{tabular}{|c|c|c|c|c|}
\hline Element & $\begin{array}{r}\text { Used oil } \\
\text { samples } \\
\left.\text { (median, } \mu \mathrm{g} \mathrm{L}^{-1}\right)\end{array}$ & $\begin{array}{r}\text { Unused oil } \\
\text { sample }\left(\mu \mathrm{g} \mathrm{L}^{-1)}\right.\end{array}$ & CF & $\begin{array}{r}\text { Fuel } \\
\text { samples } \\
\left(\text { median, } \mu \mathrm{L} \mathrm{L}^{-1}\right)\end{array}$ \\
\hline $\mathrm{Pb}$ & $1,453,147.68$ & 333.99 & $4,350.86$ & $495,632.69$ \\
\hline $\mathrm{Na}$ & $74,717.48$ & $67,344.61$ & 1.11 & $10,802.29$ \\
\hline $\mathrm{P}$ & $1,002,307.76$ & $1,207,561.00$ & 0.83 & $7,069.05$ \\
\hline $\mathrm{Zn}$ & $25,147.14$ & $13,410.86$ & 1.88 & $5,264.24$ \\
\hline $\mathrm{Si}$ & $50,144.73$ & $35,496.20$ & 1.41 & $4,063.17$ \\
\hline $\mathrm{Ca}$ & $118,571.64$ & $58,921.49$ & 2.01 & $3,843.32$ \\
\hline K & 7,169.71 & $5,967.58$ & 1.20 & $2,346.20$ \\
\hline $\mathrm{Mg}$ & $8,431.83$ & $4,825.19$ & 1.75 & 897.65 \\
\hline $\mathrm{Al}$ & $14,268.60$ & $4,652.38$ & 3.07 & 693.74 \\
\hline $\mathrm{Fe}$ & $10,435.84$ & $1,610.10$ & 6.48 & 685.46 \\
\hline $\mathrm{Cd}$ & $1,991.15$ & 4.01 & 496.97 & 267.08 \\
\hline B & 230.51 & 307.01 & 0.75 & 123.24 \\
\hline $\mathrm{Cu}$ & $7,178.34$ & 26.87 & 267.17 & 103.82 \\
\hline $\mathrm{Ni}$ & $1,029.71$ & 75.30 & 13.67 & 37.92 \\
\hline $\mathrm{Ba}$ & 36.91 & 15.04 & 2.45 & 32.93 \\
\hline $\mathrm{Mn}$ & 286.31 & 87.48 & 3.27 & 21.70 \\
\hline $\mathrm{Sr}$ & 351.87 & 175.48 & 2.01 & 16.00 \\
\hline $\mathrm{Cr}$ & $1,056.59$ & 91.29 & 11.57 & 15.25 \\
\hline $\mathrm{Ti}$ & 170.33 & 91.41 & 1.86 & 11.03 \\
\hline W & 39.44 & 22.28 & 1.77 & 8.69 \\
\hline $\mathrm{Zr}$ & 16.39 & 12.97 & 1.26 & 6.08 \\
\hline $\mathrm{Nb}$ & 4.00 & 1.40 & 2.84 & 4.83 \\
\hline Sn & 596.03 & 166.89 & 3.57 & 4.63 \\
\hline $\mathrm{Bi}$ & 28.64 & 6.47 & 4.43 & 4.32 \\
\hline $\mathrm{Ta}$ & 30.48 & 26.19 & 1.16 & 3.97 \\
\hline Mo & 67.06 & 2.65 & 25.35 & 3.75 \\
\hline $\mathrm{Hg}$ & 20.82 & 12.71 & 1.64 & 3.32 \\
\hline As & 13.85 & 8.26 & 1.68 & 2.55 \\
\hline $\mathrm{Li}$ & 28.21 & - & - & 2.16 \\
\hline $\mathrm{Rb}$ & 11.39 & 10.12 & 1.13 & 1.93 \\
\hline $\mathrm{Se}$ & 11.06 & - & - & 1.81 \\
\hline Co & 7.94 & 2.79 & 2.84 & 1.78 \\
\hline $\mathrm{Ce}$ & 4.70 & 10.72 & 0.44 & 1.34 \\
\hline $\mathrm{Ga}$ & 2.82 & 4.69 & 0.60 & 1.33 \\
\hline V & 10.25 & 13.23 & 0.78 & 1.30 \\
\hline $\mathrm{Sb}$ & 3.41 & 6.47 & 0.53 & 1.28 \\
\hline $\mathrm{Hf}$ & 0.61 & 0.76 & 0.80 & 1.23 \\
\hline $\mathrm{Be}$ & 3.46 & 2.60 & 1.33 & 1.20 \\
\hline $\mathrm{Ag}$ & 4.77 & 3.75 & 1.27 & 1.16 \\
\hline $\mathrm{Tl}$ & 1.38 & 0.58 & 2.37 & 1.15 \\
\hline Sc & 3.77 & 8.03 & 0.47 & 1.10 \\
\hline Th & 1.53 & 1.37 & 1.12 & 1.03 \\
\hline $\mathrm{Nd}$ & 2.37 & 1.68 & 1.41 & 0.80 \\
\hline $\mathrm{Te}$ & 1.57 & 4.75 & 0.33 & 0.65 \\
\hline Cs & 0.91 & 1.41 & 0.64 & 0.59 \\
\hline $\mathrm{La}$ & 2.69 & 5.83 & 0.46 & 0.57 \\
\hline Y & 2.92 & 5.23 & 0.56 & 0.43 \\
\hline $\mathrm{Ge}$ & 2.57 & 3.94 & 0.65 & 0.29 \\
\hline $\mathrm{Gd}$ & 0.62 & 0.76 & 0.81 & 0.23 \\
\hline $\mathrm{Pr}$ & 0.75 & 0.63 & 1.19 & 0.23 \\
\hline $\mathrm{U}$ & 1.82 & 1.13 & 1.61 & 0.20 \\
\hline In & 2.26 & 0.52 & 4.33 & 0.14 \\
\hline Dy & 0.42 & 0.65 & 0.65 & 0.08 \\
\hline $\mathrm{Sm}$ & 0.43 & 0.69 & 0.62 & 0.07 \\
\hline $\mathrm{Yb}$ & 0.20 & 0.24 & 0.84 & $<0.05$ \\
\hline Er & 0.20 & 0.26 & 0.80 & $<0.05$ \\
\hline $\mathrm{Eu}$ & 0.10 & 0.14 & 0.74 & $<0.05$ \\
\hline Ho & 0.07 & 0.09 & 0.81 & $<0.05$ \\
\hline $\mathrm{Tb}$ & 0.09 & 0.10 & 0.91 & $<0.05$ \\
\hline $\operatorname{Tm}$ & $<0.05$ & $<0.05$ & 3.73 & $<0.05$ \\
\hline $\mathrm{Lu}$ & $<0.05$ & $<0.05$ & 0.81 & $<0.05$ \\
\hline
\end{tabular}

